This is an electronic reprint of the original article. This reprint may differ from the original in pagination and typographic detail.

\author{
Author(s): Tanskanen, Minna; Uusitalo, Arja; Kinnunen, Hannu; Häkkinen, Keijo; Kyröläinen, \\ Heikki; Atalay, Mustafa
}

Title: $\quad$ Association of military training with oxidative stress and overreaching

Year: $\quad 2011$

Version:

Please cite the original version:

Tanskanen, M., Uusitalo, A., Kinnunen, H., Häkkinen, K., Kyröläinen, H., \& Atalay, M. (2011). Association of military training with oxidative stress and overreaching. Medicine \& Science in Sports \& Exercise, 43(8), 1552-1560..

https://doi.org/10.1249/MSS.0b013e3182106d81

All material supplied via JYX is protected by copyright and other intellectual property rights, and duplication or sale of all or part of any of the repository collections is not permitted, except that material may be duplicated by you for your research use or educational purposes in electronic or print form. You must obtain permission for any other use. Electronic or print copies may not be offered, whether for sale or otherwise to anyone who is not an authorised user. 


\section{Association of military training with oxidative stress and overreaching}

Minna M. Tanskanen ${ }^{1}$, Arja L. Uusitalo ${ }^{2}$, Hannu Kinnunen ${ }^{3}$, Keijo Häkkinen ${ }^{1}$, Heikki Kyröläinen ${ }^{1}$, Mustafa Atalay ${ }^{4}$

${ }^{1}$ Department of Biology of Physical Activity, University of Jyväskylä, Finland

${ }^{2}$ Department of Clinical Physiology and Nuclear Medicine, Helsinki University Hospital, Finland

${ }^{3}$ Polar Electro Oy, Kempele, Finland

${ }^{4}$ Institute of Biomedicine, Physiology, University of Eastern Finland, Kuopio, Finland

\section{Corresponding author:}

Minna Tanskanen

University of Jyväskylä, Department of Biology of Physical Activity

Kidekuja 2, Snowpolis

88610 VUOKATTI

FINLAND

tel. +358 505948 564, fax. +358 8617 8641, e-mail minna.m.tanskanen@jyu.fi

Running title: Oxidative stress and overreaching

Disclosure of funding: The study was granted by the Finnish Ministry of Education, Finnish Cultural Foundation, Polar Electro Oy and the Scientific Advisory Board for Defence. 


\section{ABSTRACT}

We hypothesized that increased oxidative stress and disrupted redox balance may be predisposing factors and markers for overreaching (OR). Purpose: To examine whether oxidative stress markers and antioxidant status and physical fitness are related to overreaching during an 8-week military basic training (BT) period. Methods: Oxidative stress and antioxidant status were evaluated in the beginning, after 4 and 7 weeks of training in 35 males (age $19.7 \pm$ 0.3 yrs) at rest and immediately after a 45-min submaximal exercise. Physical activity (PA) was monitored by accelerometer throughout BT. Indicators of OR were also examined. Results: From baseline to wk4 increased daytime moderate to vigorous PA led to concomitant decreases in the ratio oxidized to total glutathione (GSSG/TGSH) and GSSG. After 4 weeks of BT, GSSG/TGSH and GSSG returned to the baseline values at rest, while PA remained unchanged. At every time point acute exercise decreased TGSH and increased GSSG and GSSG/TGSH, while a decrease was observed in antioxidant capacity after 4 weeks of training. In the beginning of BT, OR subjects (11 of the 35 males) had higher GSSG, GSSG/TGSH and malondialdehyde (a marker of lipid peroxidation) at rest $(\mathrm{P}<0.01-0.05)$ and lower response of GSSG and GSSG/TGSH ratio $(\mathrm{P}<0.01)$ to exercise than noOR subjects. Moreover, OR subjects had higher PA during BT than noOR $(\mathrm{P}<0.05)$. Conclusion: The sustained training load during the last four weeks of BT led to oxidative stress observable both at rest and after submaximal exercise. Increased oxidative stress may be a marker of insufficient recovery leading possibly to OR.

Keywords: training load; physical activity; exercise testing; military; male; young adult 


\section{INTRODUCTION}

Paragraph 1. In competitive sports and military training the goal of the training is to improve performance. However, both prolonged aerobic and strength training with high intensity or volume together with inadequate recovery could lead to non-functional overreaching (OR) resulting in stagnation or decrements in performance capacity. (16). OR could also lead to the development of OTS, both of which are characterized by an unexpected decrease or at least stagnation in performance despite increased or sustained training load. The time required for the recovery from OR takes several days to several weeks, whereas the development of OTS and recovery from it may take much longer time.

Paragraph 2. Several factors and symptoms have been linked to OTS. However, there are no reliable and specific markers of impending OTS, nor knowledge on how to identify individuals who are more vulnerable to OR. Moreover, the precise mechanisms leading to OR and OTS have not yet been delineated. Recently, impaired antioxidant capacity and increased oxidative stress, a state in which the production of reactive oxygen species (ROS) overwhelms antioxidant defenses, was found to be related to OTS in elite athletes $(28)$. Animal $(18,36)$ and human studies have provided evidence that a progressively increased training volume, which causes symptoms of OR or OTS, can increase oxidative stress and attenuate antioxidant capacity (15, 35). While a single bout of physical exercise of sufficient intensity and duration generate ROS, both aerobic (21) and anaerobic training (2) have been shown to enhance antioxidant status and decrease the generation of ROS. In contrast, overload training can lead to an impaired antioxidant defense and lack of expected adaptations to training (25), and distortion of the redox balance (8). Extreme increases in training volumes may lead to substantial rise in inflammation 
and apoptosis markers (7). Therefore, overload training may induce inflammation, which is also associated with increased oxidative stress.

Paragraph 3. We hypothesized that increased oxidative stress and disrupted redox balance in response to heavy physical training may be predisposing factors and markers for overreaching (OR). For this purpose we aimed to study the association of oxidative stress markers and antioxidant status with physical activity during an 8-week military basic training (BT) period. BT comprises the first eight weeks of military service and includes both endurance and strength type of training. A particular aim was to evaluate whether the levels of oxidative stress markers and antioxidant status and physical activity differ between OR and noOR subjects.

\section{METHODS}

Paragraph 4. Subjects. Male conscripts $(\mathrm{N}=35$, aged $19.6 \pm 0.3$ yrs $)$ participated in the present study. They were a part of a larger group of 60 soldiers who initially volunteered to participate and fulfilled selection criteria (29). All subjects were fully informed of the experimental protocol, and gave their written consent to participate in the study. They were also advised of their right to withdraw from the investigation at any time. The study protocol was approved by the Finnish Defence Forces, and the Ethical Committees of the University of Jyväskylä and the Kainuu region of Finland. The study took place during winter in Finland, when daily outdoor temperatures ranged from $-31^{\circ} \mathrm{C}$ to $+1^{\circ} \mathrm{C}$, with an average of $-13^{\circ} \mathrm{C}$ (data from the local weather station). "The overall physical load of the 8-week BT period was set according to the standard basic program (31). It included a total of 300 hours military training, of which 100 hours was military related physical training, 33 hours sports related physical training such as combat 
training and marching, and the rest of hours were other military training such as shooting, material handling, skill training and general military education (23). The intensity level of physical activity in the daily program was planned to be low in the first week of BT and increased gradually thereafter. The conscripts slept from 10 p.m. to 5:45 a.m. in dormitory-type rooms at the garrison and marched four times per day (approximately $5 \mathrm{~km}$ in total) for meals. The BT schedule included four longer (from two to eight hours) marching exercises with a combat gear and two overnight field exercises from one up to three days. Food and water intake were in accordance with the standard army meal, and water intake was not restricted. However, subjects were not permitted to use any extra nutritional supplements throughout the study."

Paragraph 5. Experimental protocol. Performance tests (maximal aerobic fitness $\left[\mathrm{VO}_{2} \mathrm{max}\right]$ test and submaximal exercise test) were performed and oxidative stress and antioxidant status determined three times and psychological markers (questionnaires) five times during the 8-week training period. In addition, acute responses to a submaximal exercise were studied. For the submaximal exercise, a marching test with a $20 \mathrm{~kg}$ backpack was selected because it closely resembles the routine activities of conscripts and enables the test to be performed for a large group of subjects. The experimental protocol is presented in Table 1.

Paragraph 6. Performance tests. An incremental test until voluntary exhaustion to determine maximal oxygen uptake $\left(\mathrm{VO}_{2} \mathrm{max}\right)$ was carried out on a treadmill at baseline and on weeks five (wk5) and eight (wk8) as described earlier (29). The 45-minute submaximal exercise test was performed on an outdoor track at the $70 \%$ level of subject's individual maximal workload in the beginning (baseline) of BT at baseline, on the weeks four (wk4) and seven (wk7). The follow-up tests for each subject were always performed at the same time of day $\left(\mathrm{VO}_{2}\right.$ max-test between 8.30 
a.m. and 5 p.m., submaximal exercise between 9 a.m. and 12 p.m.) having consumed a similar diet before each test with the same test protocol. The hormonal responses to submaximal exercise have previously been reported (30).

Paragraph 7. Body composition. Body composition measurements (body mass [BM], fat free mass $[\mathrm{FFM}]$, fat mass $[\mathrm{FM}]$ and percentage of body fat $[\mathrm{F} \%]$ ) were performed using eight-point bioelectrical impedance (Inbody720, Biospace Co. Ltd, Seoul, Korea). For each subject, the repeated measurements were performed at the same time between 6 and 7 a.m. after an overnight fast and after voiding, with no exercise for 12 hours before the test. The physical activities in the daily program were planned to be of a low intensity on the day preceding each measurement and fluid status was estimated to be in balance based on the dietary records of the subjects (29). The subjects were barefoot and wore T-shirts and trousers. Body height was measured to the nearest $0.5 \mathrm{~cm}$ using a wall-mounted stadiometer. Body mass index (BMI) was calculated as BM (kg) divided by height (m) squared.

Paragraph 8. Blood samples and analyses. Blood samples from an antecubital vein and fingertip were drawn 2 hours after a light breakfast at rest, before submaximal exercise, and immediately after exercise. Circadian variability in blood parameters was minimized by collecting individual pre- and post-exercise samples at the same time of day between 9 and 12 a.m. preceded by similar patterns of food ingestion. Subjects were instructed not to ingest alcohol, coffee, tea, chocolate or cola drinks since the previous evening of the measurements.

Paragraph 9. Blood samples were centrifuged at $1200 \mathrm{~g}$ and $4^{\circ} \mathrm{C}$ for $15 \mathrm{~min}$ immediately after collection to separate the plasma. Plasma samples were stored in multiple portions at $-80^{\circ} \mathrm{C}$ until 
analysis. Protein carbonyls, markers of protein oxidative damage, were measured using an ELISA method as previously described (19). Nitrotyrosine concentrations were determined with the ELISA method using a commercial kit (HyCult biotechnology b.v; Netherlands). Lipid peroxidation marker total malondialdehyde in plasma was measured according to the method of Gerard-Monnier et al. (9). Oxygen radical absorbance capacity (ORAC) was used for the measurement of antioxidant capacity, which was performed using a multi-well plate reader according to the methods described previously (13). The maximal intra-assay coefficients of variation for protein carbonyls, nitrotyrosine, malondialdehyde and ORAC were 5.9\%, 10.0\%, $6.2 \%$ and $8.1 \%$, respectively, and the maximal inter-assay coefficients of variation were $9.2 \%$, $12.7,10.6 \%$ and $11.3 \%$, respectively. Protein carbonyls and ORAC measurements were performed in triplicate. Total glutathione (TGSH) and an oxidative stress marker reduced glutathione (GSSG) concentrations were determined spectrophotometrically as described previously (24). The tissues were deproteinized with metaphosphorous acid (MPA) for TGSH and GSSG analysis. The maximal intra-assay coefficients of variance for these assays was $4.8 \%$, and inter-assay $6.4 \%$. Hemoglobin and hematocrit were analyzed using a Sysmex KX 21Nanalyzer (Sysmex Co., Kobe, Japan). Blood lactate was analyzed from fingertip blood sample using Lactate Pro ${ }^{\circledR}$-analyzer (Arkray, Japan). Submaximal exercise-induced changes in plasma volume was calculated from changes in hemoglobin and hematocrit (6) and malondialdehyde, nitrotyrosine and ORAC post exercise values are reported adjusted for these changes. Plasma protein carbonyl results were expressed in $\mathrm{nmol} \cdot \mathrm{mg}^{-1}$ protein. The BT and/or exercise induced relative changes are expressed as $\Delta \%$. 
Paragraph 10. Questionnaires and sick leave. The subjects rated how severely they experienced a list of symptoms during the last week using a five-point Likert scale; 1 = not at all, $2=$ one day, $3=2-3$ days, $4=4-5$ days, $5=6-7$ days. The somatic symptoms were subjective ratings of well-being, upper respiratory track infections, flu-like symptoms, digestive disorders and reduced appetite, musculo-skeletal, physical complaints and sleep difficulties (30). The sum of the symptoms associated with OTS was determined as the sum of the symptom scores. In addition, the question "Do you feel physically or mentally overloaded?" was asked. Sick leave was defined as an attendance / not-attendance in daily service because of illnesses or injuries that were evaluated by a physician.

Paragraph 11. Physical activity (PA). Physical activity (PA) was measured from 18 subjects with customized version of Polar AW200 Activity monitor that was worn on the non-dominant wrist. AW200 has been found useful and accurate for measurement of EE during long-term exercise (3). AW200 contains a uniaxial accelerometer. The acceleration signal is band pass filtered (0.3-3.0 Hz), and device has reduced sensitivity to repeated low-intensity hand movements. The device counts hand movements if acceleration exceeds $1.0 \mathrm{~m} \cdot \mathrm{s}^{-2}(12)$. Epoch length was set at one minute and a curvilinear equation was used to transform activity counts to metabolic equivalents ( 1 - 16 MET), which were further adjusted by body height. Among the same subjects, AW200 weekly physical activity energy expenditure correlated well $(r=0.78)$ to that obtained with doubly labeled water (unpublished data, see (29)). Daytime was determined from 6:00 a.m. to 9:00 p.m. and nightime from 9:00 p.m. to 6:00 a.m. Periods that contained no single movement during $30 \mathrm{~min}$ in daytime, or during 6 hours or more in nightime, were classified as non-wear time and excluded from analysis. PA by each minute was classifying into either (1) no activity = REST $\leq 1.0 \mathrm{MET}$, (2) very light to light activity $=$ VLPA, 1.0-3.9 MET or 
(3) moderate to vigorous physical activity $=$ MVPA $\geq 4$ MET. REST (during daytime) or sleep (during nighttime) was selected if accelerometer showed no hand movements within more than $50 \%$ of a 10 minute moving window. Each subject's daily and nightly data were included for analysis only if activity recording covered more than $80 \%$ of daytime and $90 \%$ of nighttime. Measures of PA were adjusted by the proportion of time recorded. The activity watches were collected every evening between 9 to 10 p.m. for data download, and redistributed within 30 minutes. Out of a total of 41 days, we included days and nights when at least two thirds of subjects had enough data. In all, 33 days and 28 nights were included in the analysis. Data were pooled into two week periods; weeks 1-2, weeks 3-4, weeks 5-6 and weeks 7-8.

Paragraph 12. Criteria for overreaching (OR). In this study subjects had to fulfill three of five criteria to be classified as OR subjects (30). Criteria 1; A reduced maximal aerobic fitness $\left(\mathrm{VO}_{2} \max \right)$ of greater than $5 \%(11,26)$ or did not perform the test because of illness. Absence from the test was set as an additional criterion, because all three $\mathrm{VO}_{2}$ max tests were completed by a total of 21 of 35 conscripts. It was assumed that illness itself reduces performance. Furthermore, overload training has been reported as a risk factor for upper respiratory track infections $(27,34)$. Criteria 2; An increase in mean RPE during the submaximal exercise greater than 1.0 (11) from the lowest value at wk1 or wk4 until the end of BT. Criteria 3; An increase in somatic symptoms of OTS $(16,32)$ greater than $15 \%$ from wk4 to $w k 7$, and remaining the same or increasing from wk7 to wk8. Subjects were divided into tertiles based on an increase in somatic symptoms of OTS from wk4 to wk7; $15 \%$ was the cut-off for the upper third. Criteria 4; Admitted feeling physically or mentally overloaded $(16,33,34)$ at week 7 or 8 . Criteria 5; Sick leave more that $10 \%$ of daily service. Subjects were divided into tertiles based on sick leave during BT; $10 \%$ was the cut-off for the upper third. 
Paragraph 13. Statistical Analyses. Statistical analyses were performed using SPSS (Version 16.0.1. 2005; SPSS Inc., Chicago, IL). The level of statistical significance was set at $\mathrm{p}<0.05$. Assumptions for normality were not met for protein carbonyls, malondialdehyde and nitrotyrosine, and data were log transformed before statistical analysis. The untransformed values are shown in the text, tables and figures for more meaningful comparison, except for nitrotyrosine. Responses to submaximal exercise (before and after) and BT period (baseline and wk4 and wk7) were assessed using repeated-measures ANOVA. Mixed-design factorial ANOVA [group (noOR vs. OR) x exercise x BT] was used to identify differences between and within the OR and noOR subjects. Bonferroni as the Post hoc analysis was used to identify significant differences. In addition, the effect of exercise and BT and their interaction were calculated. Pearson product-moment correlations were used to observe associations between variables. All data are presented as mean \pm SD.

\section{RESULTS}

Paragraph 14. $\mathrm{VO}_{2} \mathrm{max}$ and body composition. There was a main effect of $\mathrm{BT}$ for $\mathrm{VO}_{2} \max$ $(\mathrm{P}<0.001)$ with an increase in $\mathrm{VO}_{2} \max$ after 5 wk of $\mathrm{BT}(\mathrm{P}<0.001)$. From wk5 to wk8, $\mathrm{VO}_{2} \max$ did not change significantly (baseline $45 \pm 7 \mathrm{~mL} \cdot \mathrm{kg}^{-1} \cdot \mathrm{min}^{-1}$; wk4 $49 \pm 5 \mathrm{~mL} \cdot \mathrm{kg}^{-1} \cdot \mathrm{min}^{-1}$; week 7 $\left.49 \pm 5 \mathrm{~mL} \cdot \mathrm{kg}^{-1} \cdot \mathrm{min}^{-1}\right)$. The initial mean body height was $178.2 \pm 7.9 \mathrm{~cm}, \mathrm{BMI} 24.7 \pm 4.5 \mathrm{~kg} \cdot \mathrm{m}^{-2}$ and $\mathrm{F} \% 19.3 \pm 7.5 \%$. Both $\mathrm{BM}$ and $\mathrm{FM}$ exhibited a main effect of $\mathrm{BT}(\mathrm{P}<0.001)$, decreasing from wk4 to wk7 ( $<<0.001$ and $\mathrm{P}<0.01$, respectively), with lower values compared to the before BT values $(\mathrm{P}<0.001)(\mathrm{BM}$ baseline $78.7 \pm 17.7 \mathrm{~kg}$; wk4 $78.5 \pm 16.9 \mathrm{~kg}$; wk7 $77.0 \pm 15.9 \mathrm{~kg} ; \mathrm{FM}$ baseline $16.1 \pm 10.7 \mathrm{~kg}$; wk4 $15.6 \pm 10.7 \mathrm{~kg}$; wk7 $14.0 \pm 9.1 \mathrm{~kg}$ ). In contrast, the mean FFM was 
higher after 7 wk of BT compared to baseline $(\mathrm{P}<0.05)$ (baseline $62.6 \pm 9.4 \mathrm{~kg}$; wk4 $62.9 \pm 8.8$ $\mathrm{kg} ; w k 763.0 \pm 9.3 \mathrm{~kg})$.

Paragraph 15. Oxidative stress markers and antioxidant capacity. There was a main effect of exercise for TGSH $(\mathrm{P}<0.001)$, GSSG $(\mathrm{P}<0.001)$, and the GSSG/TGSH ratio $(\mathrm{P}<0.001)$, a main effect of BT for TGSH $(\mathrm{P}<0.05)$ and ORAC $(\mathrm{P}<0.01)$, and exercise $\mathrm{x}$ BT interaction for TGSH $(\mathrm{P}<0.05)$, GSSG $(\mathrm{P}<0.001)$, the GSSG/TGSH ratio $(\mathrm{P}<0.01)$ and ORAC $(\mathrm{P}<0.05)$. TGSH and ORAC (Figure 1) and protein carbonyls, malondialdehyde and nitrotyrosine at rest remained unchanged during BT. After 4 wk of BT a decrease at rest were observed in GSSG $(\mathrm{P}<0.01)$, an increase in GSSG and GSSG/TGSH ratio at the latter part of BT (Figure 1). At every time point submaximal exercise induced a decrease in TGSH and an increase in GSSG and GSSG/TGSH ratio (Figure 1). However, a significant submaximal exercise induced decrease in ORAC was observed only at wk4 (Figure 1). In addition, at wk4 TGSH and ORAC were lower after submaximal exercise than at baseline (TGSH $\mathrm{P}<0.01$; ORAC $\mathrm{P}<0.01$ ) and wk7 (TGSH $\mathrm{P}<0.001$; ORAC P<0.001). GSSG and the GSSG/TGSH ratio after submaximal exercise were higher at wk4 (GSSG $\mathrm{P}<0.05$; GSSG/TGSH $\mathrm{P}<0.01$ ) and GSSG at wk7 $(\mathrm{P}<0.001)$ than at baseline (Figure 1). No significant changes were observed due to the submaximal exercise in protein carbonyls, malondialdehyde and nitrotyrosine.

Paragraph 16. Differences between noOR and OR subjects. At least three of the five criteria were detected in $31 \%(\mathrm{~N}=11$ of 35$)$ of the subjects, all of whom were classified as OR subjects. The remaining $69 \%(\mathrm{~N}=24)$ were classified as noOR subjects. From OR subjects, nine subjects fulfilled criteria 1 , six criteria 2 , eight criteria 3 , nine criteria 4 and six subjects fulfilled criteria 
5. There was a group $\mathrm{x}$ exercise $\mathrm{x}$ BT interaction only for GSSG $(\mathrm{P}<0.001)$ and GSSG/TGSH ratio $(\mathrm{P}<0.05)$. At baseline, GSSG $(\mathrm{P}<0.01)$ and the GSSG/TGSH ratio $(\mathrm{P}<0.05)$ was higher in OR than in noOR at rest (Figures $2 \mathrm{a}$ and $2 \mathrm{c}$, respectively). In OR, no response of GSSG and GSSG/TGSH to acute submaximal exercise was seen, in contrast to noOR subjects (Figures $2 \mathrm{~b}$ and $2 \mathrm{~d}$, respectively). Furthermore, OR subjects had higher malondialdehyde at baseline $(\mathrm{P}<0.05)$ than no OR (Table 2). $\mathrm{VO}_{2} \mathrm{max}$ and body composition did not differ between the groups.

Paragraph 17. In response to $\mathrm{BT}$ at rest, OR subjects exhibited decreased GSSG $(\mathrm{P}<0.01)$ and a trend of decreased GSSG/TGSH ratio $(\mathrm{P}=0.058)$ from baseline to wk4 (Figures $2 \mathrm{a}$ and $2 \mathrm{c}$ ). Compared to baseline OR had a higher relative increase in GSSG due to submaximal exercise both after $4(\mathrm{P}<0.01)$ and $7(\mathrm{P}<0.05)$ wk of $\mathrm{BT}$, and in the GSSG/TGSH ratio after 4 wk of BT $(\mathrm{P}<0.05)$ (Figures $2 \mathrm{~b}$ and $2 \mathrm{~d}$ ). Among the noOR subjects exercise induced decrease in TGSH was lower after 7 wk of BT than after 4 wk of BT $(\mathrm{P}<0.01)$ (Table 2$)$. However, only the OR subjects exhibited a significant exercise-induced decrease in ORAC after 4 wk of BT $(\mathrm{P}<0.05)$, which was significantly lower compared to wk7 $(\mathrm{P}<0.05)$ (Figure $2 \mathrm{f})$. There were no differences between the groups either neither in TGSH, ORAC, protein carbonyls, nitrotyrosine at rest, nor in the relative responses to exercise at baseline or during BT (Table 2). The relative changes at rest during BT in TSGH, GSSG, GSSG/TGSH ratio, ORAC, protein carbonyls, malondialdehyde or nitrotyrosine also did not differ between the groups. However, OR had a higher incidence of sick leave only during the last week of BT compared to the noOR subjects (mean difference $5.5 \%, 95 \%$ CI 1.8 to $9.0 \%, \mathrm{P}<0.004)$. 
Paragraph 18. Physical activity. During the entire BT period, the average daytime MVPA was 2:07 \pm 0:24 h:min, LVPA 11:36 \pm 0:30 h:min and REST 0:30 \pm 0:18 h:min. During the nighttime between 9:00 p.m. and 6:00 a.m., the average MVPA was 0:05 \pm 0:02 h:min, VLPA 1:00 \pm 0:18 h:min and sleeping time 7:30 \pm 0:18 h:min. There was a main effect of BT for daytime MVPA $(\mathrm{P}<0.001)$, VLPA $(\mathrm{P}<0.05)$, REST $(\mathrm{P}<0.001)$ and nighttime MVPA $(\mathrm{P}<0.001)$. Daytime MVPA was higher $(\mathrm{P}<0.001)$ (Figure 3) and VLPA $(\mathrm{P}<0.05-0.001)$ (Figure 3) and REST lower $(\mathrm{P}<0.001)$ during the latter part of BT compared to the weeks 1-2 (REST; weeks 1-2 2:00 \pm 0:06 h:min; weeks 3-4 0:24 \pm 0:12 h:min weeks 5-6 0:30 \pm 0:24 h:min; weeks 7-8 0:30 \pm 0:24 h:min). However, nighttime MVPA was higher during the first four weeks of BT compared to the latter half of BT $(\mathrm{P}<0.05-0.01)($ Figure 3$)$. Nighttime VLPA and sleeping time remained the same during the entire BT.

Paragraph 19. Physical activity among noOR and OR subjects. PA was monitored from 9 OR and 9 noOR subjects. These subjects did not differ in incidence of sick leave during BT, thus there were no need to take account the influence of sick leave on physical activity. There were main effect of group for daytime VLPA $(\mathrm{P}<0.05)$ and nighttime MVPA $(\mathrm{P}<0.05)$. OR had higher nighttime MVPA compared to noOR during the weeks 3-4 $(\mathrm{P}<0.05)$ and weeks 5-6 $(\mathrm{P}<0.05)$ (Figure 3) and less daytime VLPA during weeks 1-2 ( $<<0.05)$ and weeks 5-6 $(\mathrm{P}<0.05)$ (Figure 3). However, OR had higher daytime MVPA compared to noOR during the weeks $1-2(\mathrm{P}<0.05)$ and weeks 5-6 $(\mathrm{P}<0.05)$ (Figure 3). There were no differences between the groups in REST, nighttime VLPA and sleeping time. However, REST was less during weeks 5-6 than weeks 7-8 ( $\mathrm{P}<0.01$; weeks 5-6 0:36 \pm 0:24 h:min; weeks 7-8 0:42 \pm 0:24 h:min, ) in OR, but not in noOR. 


\section{DISCUSSION}

Paragraph 20. In this study we showed that increased oxidative stress is associated with overreaching. To our knowledge, this is the first study showing that increased oxidative stress prior to the strenuous 8-week combined aerobic and strength training period was related to the incidence of OR during the training period. Oxidative stress markers, including GSSG, the GSSG/TGSH ratio and malondialdehyde at rest, were higher among OR subjects than in noOR. In addition, OR subjects had no increase in GSSG and GSSG/TGSH ratio in the submaximal exercise, which might be a consequence of high resting levels. Recently we have reported that impaired antioxidant capacity and increased oxidative stress at the state of OTS (28). However, whether increased oxidative stress is a cause for OTS or an outcome of training protocol with inadequate recovery remains unclear.

Paragraph 21. The average training load during 8-week BT was two hours per day with only half an hour of total rest during the daytime. Otherwise the day included low physical activity from sitting and standing to slow walking. This kind of high training load could be a risk factor for OR for subjects with a low fitness level. Surprisingly, OR and noOR subjects did not differ from each other according to $\mathrm{VO}_{2} \max$ or body composition at the beginning of BT. This suggests that fitness or fatness in this study are not strong underlying factors for overtraining. On the other hand, OR were more physically active both during the day and night time than noOR. These results support the hypothesis that the consequences of physical training are not only influenced by the intensity and duration of training, but also by the duration of recovery time. Among the OR subjects high physical activity during the day- and nighttime increased the accumulated training load at the end of BT, which may have contributed to the symptoms and indicators of OR. However, the OR and noOR subjects did not differ regarding to tasks of the 
military service. Thus higher physical activity might more likely be an individual property rather than an external factor.

Paragraph 22. Classification of OR/OTS is challenging, and it is rarely classified by a single criterion. In this study, OR subjects had to fulfill three of five criteria, including a decrease in aerobic physical performance. Absence from $\mathrm{VO}_{2}$ max tests or submaximal exercise was set as an additional criterion for a decreased performance. In $71 \%$ of the cases the main reason for sick leave during BT was an upper respiratory track infection (URTI). Sick leave itself could affect training responses, even resulting in a detraining effect. However, OR and noOR did not differ according to sick leave during the first 7 weeks of BT. Thus, the decrease in performance probably was mainly caused by an excessive training load. Furthermore, we have recently reported that these OR subjects had higher basal sex-hormone-binding-globulin (SHBG) than noOR subjects at baseline and also after 4 and 7 weeks of training (30). In addition, OR subjects had higher basal serum cortisol than noOR subjects after 7 weeks of training (30). Moreover, OR subject had a decrease in the testosterone/cortisol ratio at rest from wk4 to $w \mathrm{k} 7$ and the maximal $\mathrm{La} / \mathrm{RPE}$ ratio in the VO2max test from baseline to the end of $\mathrm{BT}$, while noOR subjects did not (30).

Paragraph 23. In this study, day activity time increased and rest time decreased during the first 4 weeks of BT, with a concomitant increase in aerobic performance, while body mass remained the same. Among all 35 subjects, decreased oxidative stress at rest, evident as lower glutathione oxidation (GSSG), was observed after the first four weeks of training. The decreased oxidative damage can be explained either by attenuated generation of ROS (caloric restriction) or by enhancement of tissue protection and antioxidant systems, as a consequence of adaptation to 
regular exposure to a small amount of ROS (such as from exercise) (20). Aerobic training itself has been found to decrease oxidative stress at rest also without changes in body mass (5). Thus, the decreased oxidative stress observed in this study may be a consequence of an enhanced antioxidant defense system in response to a tolerable training load $(10,14)$, also among OR subjects. The high capacity to consume oxygen (enhanced $\mathrm{VO}_{2} \mathrm{max}$ ) and consequently to produce a larger amount of ROS are accompanied by high erythrocyte glutathione peroxidase activity and high glutathione concentration which both serve to protect the organism from lipid peroxidation and cell membrane damage (14).

Paragraph 24. In contrast to the decreased oxidative stress at rest, four weeks of BT increased the susceptibility to acute exercise-induced oxidative stress evident as higher glutathione oxidation and lower antioxidant capacity after a submaximal exercise. At baseline the subjects were not familiar with an acute physical load that may lead to increased oxidative stress. In contrast we observed a higher exercise-induced oxidative stress at wk4 than at baseline in the trained subjects. In previous studies both aerobic (8) and strength (17) training has been found to reduce post-exercise oxidative stress at the same physical task. However, Vollaard et al. (35) found that neither tapering nor overload endurance training affected the exercise-induced increase in GSSG among endurance-trained athletes. Our results may indicate overreaching due the intensive BT period. In the entire group of 35 subjects and in the OR subjects a U-shape curve of oxidative stress at rest was observed during BT; GSSG decreased at rest from baseline to week 4, increasing back to baseline values at week 7 . Interestingly, submaximal exercise decreased ORAC at wk4. This might be explained by the increased consumption of body's endogenous antioxidant resources. While coping with oxidative stress, plasma antioxidants are mobilized into the tissue (15). 
Paragraph 25. During the second half of the 8-week training period, although day activity time and rest time remained the same, GSSG and the GSSG/TGSH ratio at rest increased and submaximal exercise resulted in a higher induction of GSSG/TGSH ratio. Also stagnation in $\mathrm{VO}_{2} \mathrm{max}$ was observed and all the subjects had a decrease in fat mass. All these findings indicate that that training load was too strenuous during the second half of BT causing oxidative stress $(10,15)$. However, ORAC did not response to submaximal exercise at the end of BT, as was the case at wk4. This could be a marker of OR, since diminished antioxidant protection has been observed among overtrained athletes (28).

Paragraph 26. Plasma nitrotyrosine and protein carbonyls, markers of oxidative protein modification, remained the same during the entire 8-week training period, both at rest and after exercise. There were also no differences between the OR and noOR subjects. Plasma nitrotyrosine levels had a wide variation, which may partly explain the observed findings. The present results also support the previous findings that plasma nitrotyrosine level may not be a sufficiently sensitive marker to assess training or acute exercise-induced changes in oxidative stress $(22,28)$. Proteins are an important target for oxidative challenge. Reactive oxygen species modify amino acid side chains of proteins to form protein carbonyls (1). Serum protein carbonyls have been found to increase after an intensive 12-week resistance training period (15), decrease after exhaustive marches (50 and $80 \mathrm{~kg}$ ) in well trained soldiers (4) and increase after an exercise test to exhaustion in athletes (28). The turnover time for protein carbonyls varies from many hours to days (4). The 8-week training period might have been too short and submaximal exercise load in the present study may not be strenuous enough to modify proteins. Exercise may also have activated a mechanism that removes the oxidatively modified proteins from the 
circulation, or alternatively, activation of antioxidant mechanisms that remove the ROS (4). In addition, differences among studies in oxidative stress responses to training and exercise may be explained by the differences in energy availability, training mode and length of the training period, fitness levels and genetic background of the subjects. The subjects in the present study were low to moderately fit conscripts in the compulsory army, and training consisted of both strength and endurance training.

Paragraph 27. In conclusion, the present results suggest that increased oxidative stress may be associated with overreaching. Although the training had favorable effects on oxidative stress markers during the first 4 weeks, sustained heavy training blunted this effect at the latter part of BT. At the end of the study military training increased oxidative stress at rest back to pre-training levels, and beyond the pre-training levels in response to submaximal exercise. Therefore, we may speculate that oxidative stress may also be a marker of insufficient recovery resulting in overreaching. Since one third of the subjects were classified as overreached, the variability of the BT program has to be taken into careful consideration. Especially, the program should involve more recovery phases, both daily and periodically.

Acknowledgements: The authors thank the conscripts who participated in this study and the staff of the Brigade of Kajaani. We express our special thanks to Ms. Taija Vaarala for her technical assistance in the blood analysis, Ms. Elina Kokkonen for her assistance in the statistical analysis, and Dr. David Laaksonen for editing the language. The authors have no conflict of interest to disclose. The results of this study do not constitute endorsement by the American College of Sports Medicine. 


\section{REFERENCES}

1. Atalay M, Laaksonen DE. Diabetes, oxidative stress and physical exercise. Journal of Sports Science and Medicine. 2002;1:1-14.

2. Bloomer RJ, Goldfarb AH. Anaerobic exercise and oxidative stress: a review. Can J Appl Physiol. 2004;29(3):245-63.

3. Brugniaux JV, Niva A, Pulkkinen I, Laukkanen RM, Richalet JP, Pichon AP. Polar Activity Watch 200: a new device to accurately assess energy expenditure. $\mathrm{Br} J$ Sports Med. 2008;44(4):245-9.

4. Chevion S, Moran DS, Heled Y, Shani Y, Regev G, Abbou B, Berenshtein E, Stadtman ER, Epstein Y. Plasma antioxidant status and cell injury after severe physical exercise. Proc Natl Acad Sci U S A. 2003;100(9):5119-23.

5. Devries MC, Hamadeh MJ, Glover AW, Raha S, Samjoo IA, Tarnopolsky MA. Endurance training without weight loss lowers systemic, but not muscle, oxidative stress with no effect on inflammation in lean and obese women. Free Radic Biol Med. 2008;45(4):503-11.

6. Dill DB, Costill DL. Calculation of percentage changes in volumes of blood, plasma, and red cells in dehydration. J Appl Physiol. 1974;37(2):247-8.

7. Fatouros IG, Destouni A, Margonis K, Jamurtas AZ, Vrettou C, Kouretas D, Mastorakos G, Mitrakou A, Taxildaris K, Kanavakis E, Papassotiriou I. Cell-free plasma DNA as a novel marker of aseptic inflammation severity related to exercise overtraining. Clin Chem. 2006;52(9):1820-4.

8. Finaud J, Lac G, Filaire E. Oxidative stress : relationship with exercise and training. Sports Med. 2006;36(4):327-58.

9. Gerard-Monnier D, Erdelmeier I, Regnard K, Moze-Henry N, Yadan JC, Chaudiere J. Reactions of 1-methyl-2-phenylindole with malondialdehyde and 4-hydroxyalkenals. Analytical 
applications to a colorimetric assay of lipid peroxidation. Chem Res Toxicol. 1998;11(10):117683.

10. Gomez-Cabrera MC, Domenech E, Vina J. Moderate exercise is an antioxidant: upregulation of antioxidant genes by training. Free Radic Biol Med. 2008;44(2):126-31.

11. Halson SL, Bridge MW, Meeusen R, Busschaert B, Gleeson M, Jones DA, Jeukendrup AE. Time course of performance changes and fatigue markers during intensified training in trained cyclists. J Appl Physiol. 2002;93(3):947-56.

12. Kinnunen H, Niva A, Nauha L, Miettinen J. Estimation of energy expenditure from wrist attached accelerometer signal using movement counting and filtering. Med Sci Sports Exerc. 2009;40(5 Supplement):172.

13. Kinnunen S, Hyyppa S, Lehmuskero A, Oksala N, Maenpaa P, Hanninen O, Atalay M. Oxygen radical absorbance capacity (ORAC) and exercise-induced oxidative stress in trotters. Eur J Appl Physiol. 2005;95(5-6):550-6.

14. Margaritis I, Tessier F, Richard MJ, Marconnet P. No evidence of oxidative stress after a triathlon race in highly trained competitors. Int J Sports Med. 1997;18(3):186-90.

15. Margonis K, Fatouros IG, Jamurtas AZ, Nikolaidis MG, Douroudos I, Chatzinikolaou A, Mitrakou A, Mastorakos G, Papassotiriou I, Taxildaris K, Kouretas D. Oxidative stress biomarkers responses to physical overtraining: implications for diagnosis. Free Radic Biol Med. 2007;43(6):901-10.

16. Meeusen R, Duclos M, Gleeson M, Rietjens G, Steinacker J, Urhausen A. Prevention, diagnosis and treatment of the Overtraining Syndrome. Eur J Sport Sci. 2006;6(1):1-14.

17. Nikolaidis MG, Paschalis V, Giakas G, Fatouros IG, Koutedakis Y, Kouretas D, Jamurtas AZ. Decreased blood oxidative stress after repeated muscle-damaging exercise. Med Sci Sports Exerc. 2007;39(7):1080-9. 
18. Ogonovszky H, Sasvari M, Dosek A, Berkes I, Kaneko T, Tahara S, Nakamoto H, Goto S, Radak Z. The effects of moderate, strenuous, and overtraining on oxidative stress markers and DNA repair in rat liver. Can J Appl Physiol. 2005;30(2):186-95.

19. Oksala NK, Paimela H, Alhava E, Atalay M. Heat shock preconditioning induces protein carbonylation and alters antioxidant protection in superficially injured guinea pig gastric mucosa in vitro. Digestive Diseases and Sciences. 2007;52(8):1897-905.

20. Radak Z, Chung HY, Goto S. Exercise and hormesis: oxidative stress-related adaptation for successful aging. Biogerontology. 2005;6:71-5.

21. Radak Z, Taylor AW, Ohno H, Goto S. Adaptation to exercise-induced oxidative stress: from muscle to brain. Exerc Immunol Rev. 2001;7:90-107.

22. Rush JW, Turk JR, Laughlin MH. Exercise training regulates SOD-1 and oxidative stress in porcine aortic endothelium. American Journal of Physiology. Heart and Circulatory Physiology. 2003;284(4):H1378-87.

23. Santtila M, Häkkinen K, Kraemer WJ, Kyröläinen H. Effects of basic training on acute physiological responses to a combat loaded run test. Mil Med. 2010;175(4):273-9.

24. Sen CK, Marin E, Kretzschmar M, Hanninen O. Skeletal muscle and liver glutathione homeostasis in response to training, exercise, and immobilization. $J$ Appl Physiol. 1992;73(4):1265-72.

25. Smith LL. Cytokine hypothesis of overtraining: a physiological adaptation to excessive stress? Med Sci Sports Exerc. 2000;32(2):317-31.

26. Snyder AC, Kuipers H, Cheng B, Servais R, Fransen E. Overtraining following intensified training with normal muscle glycogen. Med Sci Sports Exerc. 1995;27(7):1063-70. 
27. Spence L, Brown WJ, Pyne DB, Nissen MD, Sloots TP, McCormack JG, Locke AS, Fricker PA. Incidence, etiology, and symptomatology of upper respiratory illness in elite athletes. Med Sci Sports Exerc. 2007;39(4):577-86.

28. Tanskanen M, Atalay M, Uusitalo A. Altered oxidative stress in overtrained athletes. J Sports Sci. 2010;28(3):309-17.

29. Tanskanen M, Uusitalo AL, Häkkinen K, Nissilä J, Santtila M, Westerterp KR, Kyröläinen H. Aerobic fitness, energy balance, and body mass index are associated with training load assessed by activity energy expenditure. Scand J Med Sci Sports. 2009;19(6):871-8.

30. Tanskanen MM, Kyröläinen H, Uusitalo AL, Huovinen J, Nissilä J, Kinnunen H, Atalay M, Häkkinen K. Serum Sex Hormone-Binding Globulin and Cortisol Concentrations are Associated With Overreaching During Strenuous Military Training. J Strength Cond Res. 2010;Jun 10(Epub ahead of print):DOI: 10.1519/JSC.0b013e3181c1fa5d.

31. Training Division DC, Finnish Defence Forces. Standard Direction of Conscripts' Physical Training [in Finnish]. 2004.

32. Uusitalo AL. Overtraining; Making a Difficult Diagnosis and Implementing Targeted Treatment. The Physician and Sportsmedicine. 2001;29(5):35-50.

33. Uusitalo AL, Vanninen E, Valkonen-Korhonen M, Kuikka JT. Brain serotonin reuptake did not change during one year in overtrained athletes. Int J Sports Med. 2006;27(9):702-8.

34. Verde T, Thomas S, Shephard RJ. Potential markers of heavy training in highly trained distance runners. Br J Sports Med. 1992;26(3):167-75.

35. Vollaard NB, Cooper CE, Shearman JP. Exercise-induced oxidative stress in overload training and tapering. Med Sci Sports Exerc. 2006;38(7):1335-41.

36. Zoppi CC, Macedo DV. Overreaching-induced oxidative stress, enhanced HSP72 expression, antioxidant and oxidative enzymes downregulation. Scand J Med Sci Sports. 2008;18(1):67-76. 


\section{FIGURE LEGENDS}

FIGURE 1. Mean ( \pm SD) plasma a) TGSH, b) GSSG, c) GSSG/TGSH and d) ORAC concentrations before and after the 45-minute submaximal exercise at baseline, and at week 4 and 7 of basic training. Difference compared to: before exercise $* * * \mathrm{P}<0.001, * * \mathrm{P}<0.01$; baseline $\$ \$ \$ \mathrm{P}<0.001, \$ \$ \mathrm{P}<0.01, \$ \mathrm{P}<0.05$; week $4{ }^{\wedge \wedge \wedge} \mathrm{P}<0.001,{ }^{\wedge} \mathrm{P}<0.01 . \mathrm{N}=35$.

FIGURE 2. Mean ( \pm SD) plasma GSSG, GSSG/TGSH and ORAC concentrations at rest (a, c, e) and changes $(\%)$ due the 45 -minute submaximal exercise $(b, d, f)$ at baseline, and at week 4 and 7 of basic training among noOR $(\mathrm{N}=24)$ and $\mathrm{OR}(\mathrm{N}=11)$ subjects. Difference compared to: noOR \#\# $\mathrm{P}<0.01$, \# $\mathrm{P}<0.05$; baseline $\$ \$ \mathrm{P}<0.01, \$ \mathrm{P}<0.05$; week $4^{\wedge} \mathrm{P}<0.05$. Significant change due the exercise $* * * \mathrm{P}<0.001, * \mathrm{P}<0.05$

FIGURE 3. Mean $( \pm$ SD) a) daytime very low (VLPA) and b) moderate to vigorous (MVPA) physical activity and c) nighttime moderate to vigorous (MVPA) physical activity during the basic training period among noOR and OR classified subjects. Difference compared to no OR \# $\mathrm{P}<0.05$; weeks 1-2 \$\$ $\mathrm{P}<0.001, \$ \$ \mathrm{P}<0.01, \$ \mathrm{P}<0.05$; weeks 5-6 a, $\mathrm{P}<0.001$, , $\mathrm{d}, \mathrm{P}<0.01$, , $\mathrm{P}<0.05$. 
TABLE 1 . Experimental design during the 8-week military basic training period.

\begin{tabular}{|c|c|c|c|c|c|c|c|c|}
\hline Week & 1 & 2 & 3 & 4 & 5 & 6 & 7 & 8 \\
\hline $\mathrm{VO}_{2} \mathrm{max}$-test & $\mathrm{X}$ & & & & $\mathrm{X}$ & & & $\mathrm{X}$ \\
\hline Submaximal exercise test & $\mathrm{X}$ & & & $\mathrm{X}$ & & & $X$ & \\
\hline Body composition & $\mathrm{X}$ & & & $\mathrm{X}$ & & & $\mathrm{X}$ & \\
\hline Blood samples & $\mathrm{X}$ & & & $\mathrm{X}$ & & & $\mathrm{X}$ & \\
\hline Questionnaire & X & & & $\mathrm{X}$ & & $X$ & $\mathrm{X}$ & $\mathrm{X}$ \\
\hline
\end{tabular}


TABLE 2. TGSH and oxidative stress markers among noOR $(\mathrm{N}=24)$ and $\mathrm{OR}(\mathrm{N}=11)$ subjects at rest and relative change due to the submaximal exercise $(\Delta \%$ ex.)

\begin{tabular}{|c|c|c|c|c|c|c|c|c|}
\hline & & & Baseline & & Week 4 & & Week 7 & \\
\hline & & & Mean \pm SD & $\mathrm{P}$ & Mean \pm SD & $\mathrm{P}$ & Mean \pm SD & $P$ \\
\hline & At rest & noOR & $773 \pm 108$ & & $756 \pm 83$ & & $776 \pm 100$ & \\
\hline & $(\mu \mathrm{mol})$ & OR & $801 \pm 140$ & & $754 \pm 91$ & & $767 \pm 115$ & \\
\hline G & & noOR & $-11 \pm 11$ & $* * *$ & $-15 \pm 8$ & $* * *$ & $-8 \pm 7$ & $* * *, \wedge$ \\
\hline & 宸 & OR & $-12 \pm 7$ & $* * *$ & $-12 \pm 11$ & $* * *$ & $-6 \pm 16$ & $* * *$ \\
\hline & & noOR & $0.99 \pm 0.28$ & & $0.93 \pm 0.29$ & & $1.10 \pm 0.32$ & \\
\hline$\vec{d}$ & At rest $(\mu$ mol $)$ & OR & $1.24 \pm 0.29$ & \# & $1.11 \pm 0.32$ & & $1.11 \pm 0.16$ & \\
\hline 営 & & noOR & $3 \pm 24$ & & $7 \pm 26$ & & $0.3 \pm 23$ & \\
\hline$\sum^{\pi}$ & & OR & $-11 \pm 27$ & & $4 \pm 26$ & & $-10 \pm 21$ & \\
\hline$\infty$ & At rest & noOR & $0.09 \pm 0.02$ & & $0.09 \pm 0.02$ & & $0.09 \pm 0.02$ & \\
\hline రి & $\left(\mathrm{nmol} \cdot \mathrm{mg}^{-1}\right)$ & OR & $0.10 \pm 0.02$ & & $0.09 \pm 0.02$ & & $0.10 \pm 0.02$ & \\
\hline$\stackrel{0}{\Xi}$ & & noOR & $0.03 \pm 5$ & & $-1 \pm 4$ & & $1 \pm 4$ & \\
\hline$\stackrel{2}{2}$ & & OR & $0.32 \pm 5$ & & $1 \pm 4$ & & $1 \pm 5$ & \\
\hline & At rest & noOR & $6.53 \pm 2.31$ & & $6.56 \pm 2.18$ & & $6.53 \pm 2.18$ & \\
\hline . & (log adj.) & OR & $6.45 \pm 1.84$ & & $6.45 \pm 1.78$ & & $6.52 \pm 1.79$ & \\
\hline 룽 & & noOR & $4 \pm 15$ & & $-3 \pm 11$ & & $2 \pm 16$ & \\
\hline & & OR & $9 \pm 23$ & & $-1 \pm 11$ & & $-6 \pm 12$ & \\
\hline
\end{tabular}

Difference; compared to noOR \# $\mathrm{P}<0.05$; within the group compared to week $4^{\wedge \wedge} \mathrm{P}<0.01$. Significant change due the exercise $* * * \mathrm{P}<0.001$. 